\title{
Post-Marketing Safety Profile of Vortioxetine Using a Cluster Analysis and a Disproportionality Analysis of Global Adverse Event Reports
}

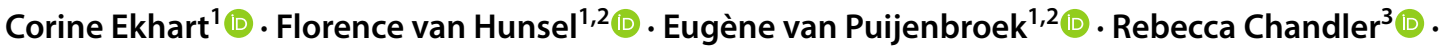 \\ Eva-Lisa Meldau ${ }^{3}$ (D) Henric Taavola ${ }^{3}\left[\right.$ - G. Niklas Norén ${ }^{3}(1)$
}

Accepted: 8 December 2021 / Published online: 12 January 2022

(c) The Author(s) 2022

\begin{abstract}
Introduction Vortioxetine, a multimodal serotonergic drug, is widely used as treatment for major depressive disorder. Although on the market since late 2013 , the data of the relative safety of vortioxetine, especially compared to selective serotonin reuptake inhibitors, are still scarce.

Objective The aim of this study was to explore the adverse event reporting pattern of vortioxetine through a cluster analysis. Furthermore, to compare the adverse event reporting pattern for vortioxetine with that of the selective serotonin reuptake inhibitors.

Methods Individual case safety reports for vortioxetine in VigiBase up to 1 November, 2019 were subjected to consensus clustering, to identify and describe natural groupings of reports based on their reported adverse events. A vigiPoint exploratory analysis compared vortioxetine to the selective serotonin reuptake inhibitors in terms of relative frequencies for a wide range of covariates, including patient sex and age, reported drugs and adverse events, and reporting country. Important differences were identified using odds ratios with adaptive statistical shrinkage.

Results Thirty-six clusters containing at least five reports were identified and analysed. The two largest clusters included $48 \%$ of the vortioxetine reports and appeared to represent gastrointestinal adverse events and hypersensitivity adverse events. Other distinct clusters were related to, respectively, fatigue, aggression/suicidality, convulsion, medication errors, arthralgia/ myalgia, increased weight, paraesthesia and anticholinergic effects. Some of these clusters are not labelled for vortioxetine, such as arthralgia/myalgia and paraesthesia, but are known adverse events for selective serotonin reuptake inhibitors. A vigiPoint analysis revealed a higher proportion of reports from consumers and non-health professionals for vortioxetine as well as higher relative reporting rates of gastrointestinal symptoms, pruritus and mood-related symptoms, consistent with the cluster analysis.

Conclusions A pattern of co-reported adverse events that is consistent with labelled adverse events for vortioxetine and the safety profile for selective serotonin reuptake inhibitors in general was revealed. Clusters of unlabelled adverse events were identified that reflect clinical entities that might represent signals of previously unknown adverse events. More extensive analyses of spontaneous reports may help to further understand the reporting pattern of adverse events.
\end{abstract}

\section{Introduction}

The information presented in this paper does not represent the opinion of the World Health Organization.

Corine Ekhart

c.ekhart@lareb.nl

1 Netherlands Pharmacovigilance Centre Lareb, Goudsbloemvallei 7, 5237 MH's-Hertogenbosch, The Netherlands

2 Groningen Research Institute of Pharmacy, Pharmacotherapy, Epidemiology and Economics, University of Groningen, Groningen, The Netherlands

3 Uppsala Monitoring Centre, Uppsala, Sweden
Vortioxetine is a novel antidepressant that was approved for the US and European markets in late 2013 [1]. It is a multimodal serotoninergic drug used to treat major depressive disorder. In addition to blockade of the serotonin transporter, vortioxetine is a full agonist at the 5-HT1A receptor and a partial agonist at the 5-HT1B receptor. It further antagonises 5-HT1D, 5-HT3 and 5-HT7 receptors (Table 1). In concert, these effects enhance levels of serotonin, norepinephrine, dopamine, acetylcholine and histamine [2]. Despite similarities with selective serotonin reuptake inhibitors (SSRIs), the mechanism of action of vortioxetine might be different from 


\section{Key Points}

By using a novel analysis method, known adverse events (e.g. gastrointestinal and hypersensitivity adverse events) as well as unknown adverse events (e.g. arthralgia/myalgia and paraesthesia) of vortioxetine have been identified.

The adverse event reporting profile of vortioxetine is generally comparable to that of the selective serotonin reuptake inhibitors; however, gastrointestinal symptoms, pruritus and mood-related symptoms were more often reported for vortioxetine than the selective serotonin reuptake inhibitors.

Novel analyses of spontaneous reports such as cluster analyses may help to understand the pathogenesis of adverse events.

SSRIs by a larger serotonin release and direct modulation of several 5-HT receptors [3] (Table 1). It is suggested that these multi-modal activities of vortioxetine have potential benefit in its safety profile and in improvement in cognition. A favourable tolerability profile for gastrointestinal effects such as nausea is expected because of its 5-HT3 antagonism [4]. Furthermore, its 5-HT1 A agonism is associated with a reduced incidence of sexual dysfunction [5]. 5-HT1B agonism is associated with low weight gain [6]. Because of its 5-HT3 and 5-HT7 antagonism, a positive effect of vortioxetine on cognition and sleep is hypothesised [4].

Several studies have evaluated the efficacy and safety profile of vortioxetine; however, there still is a lack of evidence for the relative safety and efficacy of vortioxetine, especially compared to the SSRIs. Studies with direct comparisons to SSRIs are needed to address this gap [7].

The post-marketing experience with vortioxetine is not as extensive as for SSRIs, which is reflected in the undesirable effects section of the Summary of Product Characteristics (SmPC), which is less elaborate (yet) for vortioxetine. Additionally, the amount of information found in the literature on the safety of this relatively new drug is still increasing. The most common adverse events (AEs) according to the European SmPC and the US Food and Drug Administration package insert $[8,9]$ are nausea $(\geq 10 \%)$, diarrhoea (1-10\%), constipation (1-10\%), vomiting (1-10\%), abnormal dreams (1-10\%), dizziness (1-10\%) and (generalised) pruritus (1-10\%). Furthermore, dry mouth (1-10\%) and flatulence (1-10\%) are mentioned only in the Food and Drug Administration package insert [9]. Flushing and night sweats are uncommon $(0.1-1 \%)$ and mydriasis is rare $(0.01-0.1 \%)$. Anaphylactic reaction, insomnia, serotonin syndrome,

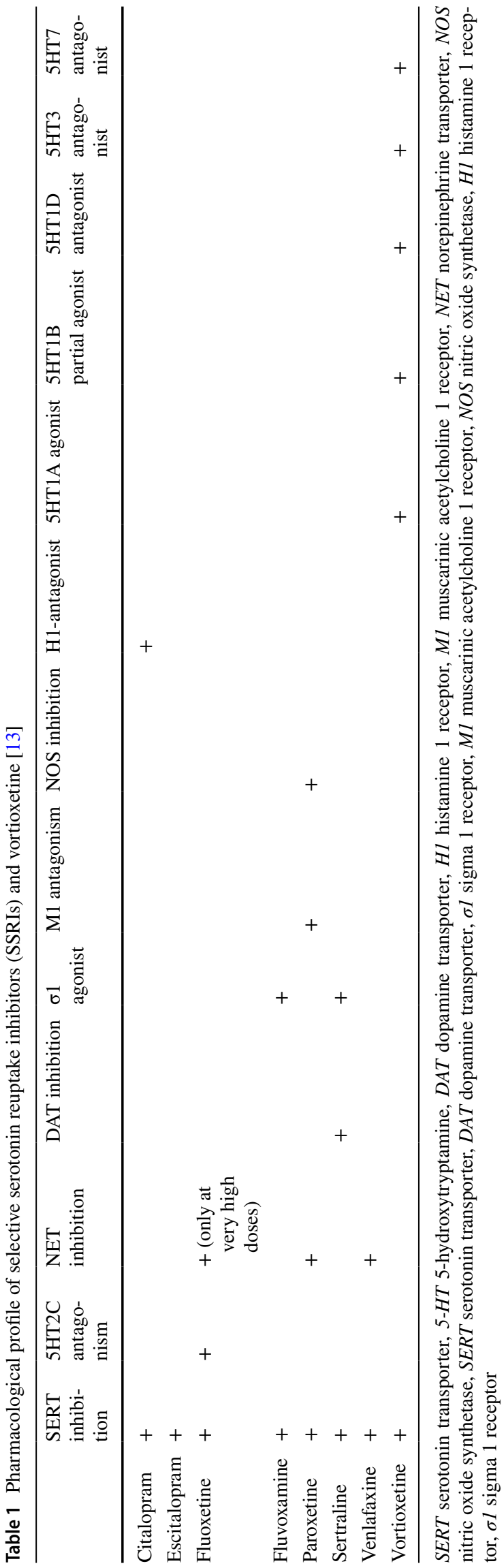


aggression/agitation, haemorrhage, hyponatraemia, angioedema, urticaria and rash have an unknown frequency as they are based on post-marketing cases [8]. Both also mention sexual dysfunction. Because of the nature of sexual dysfunction, incidences are difficult to establish $[8,9]$. The European SmPC mentions bone fractures as a possible class effect of serotonergic antidepressants [8].

Although AEs tend to be reported as distinct entities, they may share commonalities that might shed a light on the pathogenesis or an unknown underlying factor. Some AEs cannot be fully captured by single AE terms and therefore effective analyses may require the consideration of coreported AE terms. Different reporters might use different $\mathrm{AE}$ terms to describe similar conditions. However, there might be enough overlap between these reports to identify subgroups of vortioxetine reports with similar AE profiles [10]. We aim to provide insight into these subgroups of vortioxetine reports and identify these commonalities and the pathogenesis of reported AEs of vortioxetine. To reveal this overlap, a cluster analysis may be applied. This is a collection of algorithms that attempts to find common structures in a dataset and cluster the data points in the set into statistically related groups [11]. A cluster analysis seeks to identify natural subgroups in data with a closer resemblance between items within a subgroup than between items in different subgroups [12].

The aim of this article is to explore the AE reporting pattern of vortioxetine through a cluster analysis. Furthermore, to compare the $\mathrm{AE}$ reporting pattern for vortioxetine with that of the SSRIs by comparing the relative frequency of various covariates and highlighting key differences.

\section{Methods}

\subsection{VigiBase}

For this study, the World Health Organization global individual case safety report database VigiBase was used, which presently contains over 25 million reports of suspected adverse effects of medicines (June 2021), submitted since 1968 , by over 130 countries [13]. These reports are submitted by health professionals and patients and collected by national pharmacovigilance centres across the world. The information in VigiBase originates from multiple sources (different type of reporters and different countries) and the amount of information given, as well as the likelihood that a medicine caused the AE, may vary from case to case. The reported AEs are coded with the Medical Dictionary for Regulatory Activities $\left(\right.$ MedDRA $^{\circledR}$ ) and drugs are encoded with the WHODrug Global dictionary for medicinal information [14]. This database is maintained by the Uppsala Monitoring Centre in Sweden.

\subsection{AE Co-reporting Patterns}

To identify subgroups of reports with similar AE profiles, we used an AE cluster analysis algorithm grouping reports based on co-reporting patterns of AEs [12]. A cluster analysis represents a form of unsupervised learning, where the classes and their profiles are derived from data without additional guidance; in this case, from the co-reporting patterns of AEs on the reports [12]. The algorithm performs a repeated mixture-model-based cluster analysis using a latent class model for individual case reports with conditionally independent binomial distributions for the AEs. In order to increase stability, a consensus clustering groups reports that are repeatedly placed together in the mixture-model-based cluster analyses. Our implementation followed that in [12] in almost all respects. ${ }^{1}$

Our cluster analysis was implemented in Python 3.7.1. We performed cross-validation and computed the cross-validated log-likelihood that indicated that the standard hyperparameters of the algorithm gave good performance. We also computed the Adjusted Rand index to assess the stability of the obtained clusters, which was adequate.

All obtained clusters were independently reviewed by three different clinicians (CE, FvH and EvP). They determined whether clusters were probably based on the pharmacological profile, clinical scenario, or other reasons and compared the vortioxetine data with the available knowledge in the SmPC of the SSRIs.

For the cluster analysis, we retrospectively examined all AEs on vortioxetine submitted to VigiBase until 1 November, 2019. Adverse event data were analysed at the MedDRA ${ }^{\circledR}$ Preferred Term-level terminology.

\subsection{Comparison of General Reporting Patterns for Vortioxetine and SSRIs}

vigiPoint is a method for data-driven open-ended exploration in pharmacovigilance. It compares a report subset to one or more reference subsets in terms of the relative frequency of a wide range of covariates, such as patient sex and age, reported drugs and AEs, and reporting country. Non-categorical covariates, such as patient age, are divided into groups before applying the method. Each comparison (e.g. the relative frequency of reports from Sweden, or that of reports with paracetamol) is essentially univariate and independent of the other. The comparisons are

\footnotetext{
1 We included reports with two or more AE terms and we performed 100 repeated mixture-model cluster analyses, each starting with 100 initial classes and utilizing a strength of shrinkage corresponding to 50 pseudo reports. However, we required reports to have been placed together in 90 of the 100 mixture-model-based cluster analyses to be grouped in the consensus clustering (compared with 80 in Noren et al. [12]).
} 


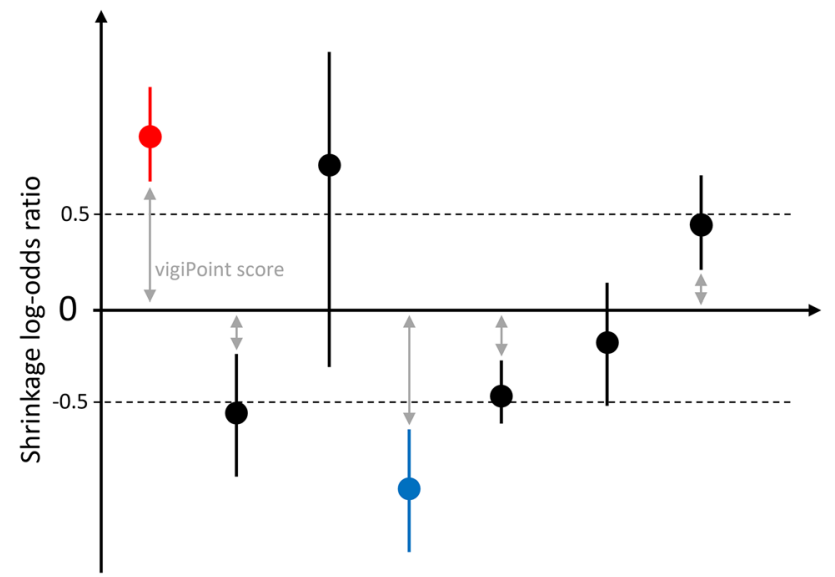

Fig. 1 Graphical explanation of the vigiPoint score

done using shrinkage log-odds ratios with $99 \%$ credibility intervals and require that the full credibility intervals be at least 0.5 above or below 0 in the $\log 2$ scale for the feature to be highlighted, which is roughly equivalent to a $40 \%$ difference in the shrunk odds ratio [15]. The distance in the log scale between 0 and the credibility interval is referred to as the vigiPoint score (Fig. 1). The shrinkage together with the high coverage credibility intervals and the 0.5 threshold ensures that only robust and relevant features are highlighted as key differences between the data subsets. Because reporting frequencies for all subgroups within all covariates are needed to calculate the vigiPoint score, these frequencies are available as output for both report subsets included in this analysis. See Fig. 1.

A vigiPoint analysis for vortioxetine vs SSRIs was carried out that investigated reports entered into VigiBase up to December 2018. For the case study, we defined the subset of interest as reports with vortioxetine as suspected or interacting, excluding reports with any of the reference drugs as suspected, concomitant or interacting. As reference reports, we used all reports with citalopram, escitalopram, fluoxetine, fluvoxamine, paroxetine, sertraline or venlafaxine as suspected or interacting, excluding reports with vortioxetine as suspected, concomitant or interacting. The covariates considered in the analysis were year of report entry into VigiBase, country of origin for the report, qualification of the reporter, patient age, patient sex, reported AEs (as MedDRA ${ }^{\circledR}$ Preferred Terms) and co-reported drugs (as WHODrug active ingredients).

\section{Results}

\subsection{AE Co-reporting Patterns}

On 1 November, 2019, there were 11,490 reports in VigiBase with vortioxetine listed as suspected or interacting.
There were 5663 vortioxetine reports with a single AE term and 5827 vortioxetine reports with multiple AE terms. A total of 2519 reports of the 5827 reports with multiple $\mathrm{AE}$ terms were included in one of the 36 clusters with five or more reports in the final consensus clustering. All 36 clusters are included in Table 1 of the Electronic Supplementary Material. Patient characteristics of the five largest clusters are shown in Table 2.

The two largest clusters comprised $48 \%$ (1197) of the vortioxetine reports included in the consensus clustering and described gastrointestinal AEs and hypersensitivity/skin reactions, respectively. These clusters were further characterised by the fact that they contained on average few AEs per report (2.7 and 2.4 respectively) (Fig. 2).

Other distinct clusters identified were related to medication errors (cluster 3), fatigue (clusters 4 and 5), aggression/ suicidality (clusters 6, 7, 10 and 12), convulsion (cluster 13), arthralgia/myalgia (cluster 8), weight increased (cluster 9), paraesthesia (cluster 14), anticholinergic effects [such as dry mouth and blurred vision] (clusters 15 and 16), and nightmares and abnormal dreams (cluster 25). This last cluster is a small cluster with only eight reports. All these eight reports contained exactly the terms abnormal dreams and nightmare.

Cluster 9 and cluster 22 are both about food craving and increased weight, although cluster 9 mentions increased weight in $82 \%$ of the cases combined with increased appetite in $51 \%$ of the cases and cluster 22 mentions hunger in $100 \%$ of the cases while $64 \%$ of the patients in this cluster have an increased weight. Cluster 27 seems related to angio-oedema, although this is not specifically mentioned in the underlying MedDRA ${ }^{\circledR}$ Preferred Terms (e.g. tongue swelling, lip swelling, eye swelling).

Fourteen clusters have fewer than ten reports (clusters 23-36).

\subsection{Comparison of General Reporting Patterns for Vortioxetine and SSRIs}

On 27 December, 2018 there was a total of 8552 vortioxetine reports that were compared with 327,478 reports of citalopram, escitalopram, fluoxetine, fluvoxamine, paroxetine, sertraline or venlafaxine in VigiBase. The first vortioxetine report as a suspect/interacting drug in VigiBase is from 2014. The reporting of vortioxetine has increased over time. There were reports from all geographical regions, but North America (70\% for vortioxetine reports, 55\% for SSRI reports) and Europe (25\% for vortioxetine reports, 34\% for SSRI reports) contributed the most. The sex distribution was the same for vortioxetine reports as for the reference reports (male $=30 \%$, female $=70 \%)$.

As highlighted by the vigiPoint analysis, vortioxetine reports were more often from consumers/non-health 
Table 2 Patient characteristics of the five largest clusters

\begin{tabular}{|c|c|c|c|c|c|c|}
\hline & \multicolumn{3}{|c|}{ Patient sex } & \multicolumn{2}{|l|}{ Patient age } & \multirow{2}{*}{$\begin{array}{l}\text { Country } \\
\text { Top reported }\end{array}$} \\
\hline & Female & Male & Unknown & $\begin{array}{l}\text { Median age } \\
\text { (years) }\end{array}$ & Unknown & \\
\hline Overall (5827 reports) & 3855 & 1518 & 454 & 47 & 2337 & USA (3631 reports) \\
\hline Cluster 1 (819 reports) & 591 & 168 & 60 & 47 & 349 & USA (476 reports) \\
\hline Cluster 2 (378 reports) & 298 & 51 & 29 & 48 & 148 & USA (214 reports) \\
\hline Cluster 3 (251 reports) & 117 & 52 & 82 & 35 & 178 & USA (230 reports) \\
\hline Cluster 4 (235 reports) & 142 & 92 & 1 & 32 & 0 & USA (235 reports) \\
\hline Cluster 5 (155 reports) & 91 & 59 & 5 & 39 & 34 & USA (126 reports) \\
\hline
\end{tabular}

Cluster 1 (819 reports)

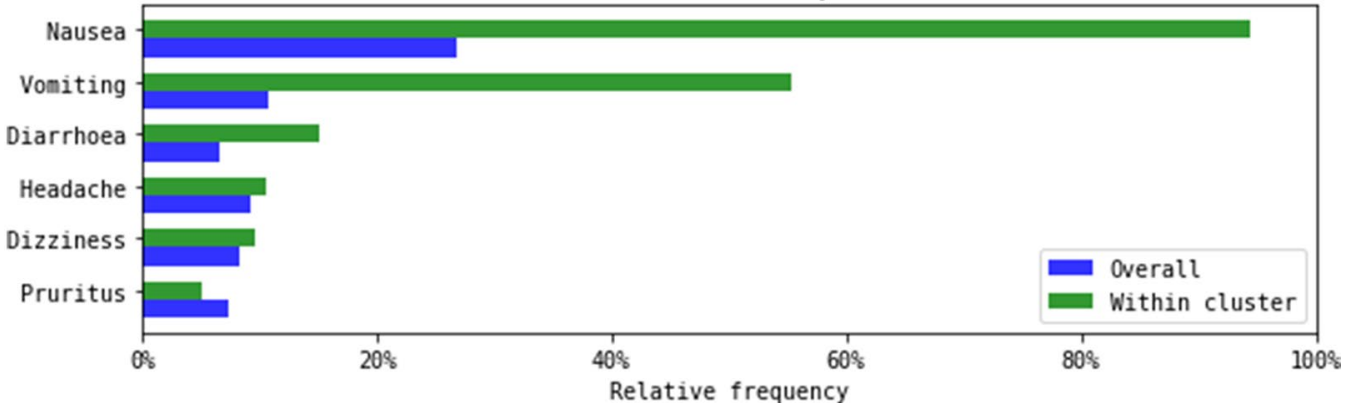

Cluster 2 (378 reports)

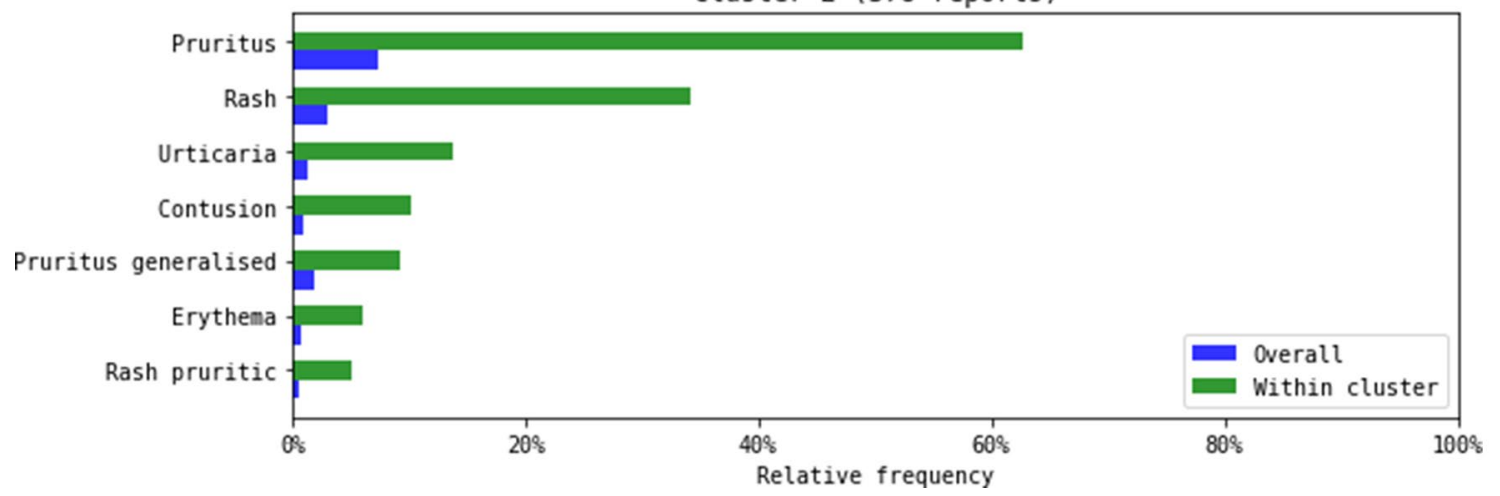

Fig. 2 Graphical presentation of adverse events in cluster 1 and cluster 2

professionals than for the SSRIs (53\% vs 29\%) and less often by physicians ( $34 \%$ vs $51 \%$ ) and pharmacists (4\% vs $10 \%$ ). Adverse events with higher and lower relative reporting rates in the vortioxetine reports compared with the SSRI reports are shown in Tables 3 and 4, respectively.

\section{Discussion}

Our analysis of global reports of suspected AEs following administration of vortioxetine revealed a diverse pattern of clusters. Some of these clusters are not labelled AEs for vortioxetine, but are known AEs for SSRIs. Some clusters can be explained by the pharmacology of vortioxetine or the pathophysiology of depression. Other clusters seem to be driven by variations in coding practice or might be spurious because of the small number of reports. For instance, for the clusters 9 and 22 about food craving and increased weight, it is difficult to entangle if these clusters are indeed about separate clinical entities or that they differ in severity or that the AEs in the reports ended up in different clusters because of differences in coding practice.

The two largest clusters identified, which contained gastrointestinal AEs and hypersensitivity AEs, are well characterised and are included in the product label $[8,9]$. Although a favourable profile for gastrointestinal effects such as nausea is expected for vortioxetine because of its 5-HT3 antagonism, it was the largest cluster identified. The 
Table 3 Adverse events identified as key features with higher relative reporting rates in the vortioxetine reports compared to the reference reports
Table 4 Adverse events identified as key features with lower relative reporting rates in the vortioxetine reports compared to the reference reports

\begin{tabular}{lllll}
\hline MedDRA ${ }^{\circledR}$ Preferred Terms & $\begin{array}{l}\text { Vortioxetine } \\
\text { reports, } \% \\
(n=8552)\end{array}$ & $\begin{array}{l}\text { SSRI reports, } \% \\
(n=327,478)\end{array}$ & Odds ratio $^{\text {a }}$ & vigiPoint score \\
\hline Pruritus & 6.7 & 1.6 & 4.5 & 1.5 \\
Nausea & 18 & 6.6 & 3.2 & 1.4 \\
Pruritus generalised & 2.3 & 0.069 & 34 & 1.4 \\
Apathy & 2.7 & 0.42 & 6.5 & 1.2 \\
Anger & 3.1 & 0.66 & 4.9 & 1.1 \\
Disturbance in attention & 3.5 & 0.91 & 4.0 & 1.1 \\
Hypersomnia & 2.0 & 0.21 & 9.5 & 1.1 \\
Hyperphagia & 1.5 & 0.022 & 68 & 1.0 \\
Feeling guilty & 1.4 & 0.060 & 24 & 0.9 \\
Asthenia & 4.4 & 1.6 & 2.8 & 0.9 \\
Irritability & 3.2 & 1.0 & 3.3 & 0.9 \\
Vomiting & 6.1 & 2.8 & 2.3 & 0.8 \\
Mood swings & 1.8 & 0.41 & 4.5 & 0.8 \\
Feeling abnormal & 4.5 & 1.9 & 2.4 & 0.8 \\
Fatigue & 5.8 & 2.8 & 2.2 & 0.7 \\
Constipation & 2.3 & 0.90 & 2.6 & 0.6 \\
Weight increased & 4.1 & 2.2 & 1.9 & 0.5 \\
Suicidal ideation & 4.4 & 2.4 & 1.9 & 0.5 \\
\hline
\end{tabular}

MedDRA medical dictionary for regulatory activities, SSRI selective serotonin reuptake inhibitor

${ }^{a}$ These are regular odds ratios and not the shrinkage log-odds ratio described in Sect. 2

\begin{tabular}{lllll}
\hline MedDRA ${ }^{\circledR}$ preferred terms & $\begin{array}{l}\text { Vortioxetine } \\
\text { reports, } \% \\
(n=8552)\end{array}$ & $\begin{array}{l}\text { SSRI reports, } \% \\
(n=327,478)\end{array}$ & Odds ratio & vigiPoint score \\
\hline Drug withdrawal syndrome & 0.11 & 4.3 & 0.023 & -1.9 \\
Exposure during pregnancy & 0.082 & 1.6 & 0.051 & -0.9 \\
Toxicity to various agents & 0.14 & 1.6 & 0.085 & -0.9 \\
Hyponatraemia & 0.53 & 2.3 & 0.23 & -0.8 \\
Foetal exposure during pregnancy & 0.047 & 1.3 & 0.036 & -0.8 \\
Withdrawal syndrome & 0.22 & 1.6 & 0.14 & -0.7 \\
Tremor & 1.5 & 3.8 & 0.39 & -0.7 \\
Paraesthesia & 0.76 & 2.4 & 0.31 & -0.7 \\
Completed suicide & 1.0 & 2.7 & 0.37 & -0.6 \\
Confusional state & 0.74 & 2.2 & 0.32 & -0.6 \\
Suicide attempt & 0.68 & 2.1 & 0.32 & -0.6 \\
Death & 0.36 & 1.5 & 0.23 & -0.6 \\
Product substitution issue & 0.023 & 0.96 & 0.024 & -0.6
\end{tabular}

MedDRA medical dictionary for regulatory affairs, SSRI selective serotonin reuptake inhibitors

${ }^{a}$ These are regular odds ratios and not the shrinkage log-odds ratio described in Sect. 2 blockade of the serotonin transporter and 5-HT1A agonism of vortioxetine might be responsible for the gastrointestinal effects seen [4]. Nausea is a known effect of drugs that block the serotonin transporter and of selective 5-HT1A receptor agonists [16].
We identified two clusters of fatigue symptoms. This is not a labelled AE. Regarding the Preferred Terms seen in this cluster, fatigue might be related to two mechanisms: psychogenic and physical (the number of co-reported Preferred Terms such as diarrhoea and hyperhidrosis is high 
[23\%]). Fatigue symptoms can also be related to the indication of vortioxetine. However, in the literature, a role of serotonin in the pathogenesis of fatigue has been suggested, as an increase in the central ratio of serotonin to dopamine is associated with feelings of tiredness and lethargy, accelerating the onset of fatigue [17].

Furthermore, aggression/suicidality clusters were identified. Vortioxetine increases serotonergic activity in the central nervous system by inhibition of neuronal reuptake of serotonin. Serotonin is supposed to have a role in the inhibition of impulses, the regulation of emotions and social functioning, which are domains linked to aggression [18]. Several mechanisms are postulated by which increased serotonergic activity might cause aggression. These include the production of feelings that often begins with lesser degrees of insomnia, nervousness, anxiety, hyperactivity, and irritability and then progress towards more severe agitation, aggression and varying degrees of mania. Another proposed mechanism is the production of a combined state of stimulation and depression (an agitated depression) with a high risk of suicide and violence. Furthermore, the production of obsessive preoccupations with aggression against self or others, often accompanied by a worsening of any preexisting depression. Finally, the production of akathisia, an inner agitation that causes heightened irritability and frustration with aggression against self or others [19]. The Uppsala Monitoring Centre and Lareb reported this association of vortioxetine and aggression in May 2019 [20]. In July 2020 , it was concluded by the committee responsible for human medicines from the European Medicines Agency that a causal relationship between vortioxetine and aggression and agitation is at least a reasonable possibility and therefore the product information of products containing vortioxetine should be amended accordingly [21]. The manufacturer has since then updated the product information $[8,9]$.

Another identified cluster was a cluster of musculoskeletal symptoms, such as arthralgia, pain in extremities and myalgia. These are no known side effects of vortioxetine and the mechanism by which vortioxetine might cause these symptoms is unknown. However, arthralgia and myalgia are labelled for SSRIs such as (es)citalopram, fluoxetine, fluvoxamine, paroxetine and sertraline [22].

Sensory disturbances such as hypoaesthesia and paraesthesia were also identified in a cluster. Serotonin has been associated with sensory disturbances. It is postulated that the sensory disturbances associated with both SSRI use and withdrawal could be due to the hyperserotonergic state. Selective serotonin reuptake inhibitor therapy increases serotonin levels in the brain acutely (though the therapeutic effect is delayed by several weeks), which could account for the early appearance of sensory symptoms, and the disappearance by 2-3 weeks may relate to homeostatic compensation [23]. Sensory disturbances are not mentioned in the product information of vortioxetine; however, they are mentioned in the product information of the SSRIs as AEs and/or withdrawal symptoms [22].

Furthermore, a cluster of anticholinergic effects such as dry mouth and blurred vision was identified. Selective serotonin reuptake inhibitor-induced dry mouth has been reported to result from inhibition of salivary secretion by blockade of the mACh receptor [24]. Selective serotonin reuptake inhibitors and serotonin and noradrenaline reuptake inhibitors may cause mydriasis by noradrenergic effects or anticholinergic effects or by 5-HT effects, which can cause relaxation of the sphincter muscle of the pupil [25]. For the SSRIs, dry mouth and visual disturbance are labelled [22]. Mydriasis is labelled for vortioxetine in the European SmPC [8].

Despite the fact that 5-HT1B agonism is associated with low weight gain [6], we identified two clusters (cluster 9 and 22) both about food craving and increased weight. Hyperphagia and weight increase were also identified as key features with higher relative reporting rates in the vortioxetine reports compared with the SSRI reports (Table 3). Weight gain during antidepressant treatment, however, can be a sign of improvement in patients whose weight loss was one of the depressive symptoms.

Although there were 16 reports of haemorrhage, it was striking to see that no cluster of haemorrhagic events was identified. An increased tendency of haemorrhagic events is a known serotonergic effect and also mentioned in the product label of vortioxetine [8]. This is probably caused by the small number of reports, combined with the lack of shared co-reported AEs between these reports.

Furthermore, because of the 5-HT1A agonism of vortioxetine, a reduced incidence of sexual dysfunction is proposed [5]. We did not identify a cluster of sexual dysfunction. However, AEs of sexual dysfunction were seen in various clusters (libido decreased [clusters 4 and 20], erectile dysfunction [cluster 9], loss of libido [cluster 22] and sexual dysfunction [cluster 33]).

In the vigiPoint analysis, we compared reports submitted for vortioxetine with the SSRIs. Venlafaxine has potency at serotonin transporters, which is about 30 -fold greater than that at norepinephrine transporters. Thus, at low doses, venlafaxine acts essentially as a SSRI, with significant noradrenergic activity only occurring at higher doses [26]. Therefore, we chose to add venlafaxine to the SSRI reference group. We did not perform a sensitivity analysis to see if the results would be different if we removed the drug from the reference group. This vigiPoint analysis highlighted that vortioxetine reports are more often reported by consumers than healthcare professionals. We found it interesting that for a relatively new drug, with limited information available in the literature and official product label, consumers report more often than healthcare professionals and thus could have 
an important contribution to signal detection for this drug. Indeed, other studies have shown that consumers can have an important contribution to the detection of safety signals [27, 28]. Adverse events such as gastrointestinal symptoms, pruritus and mood-related symptoms were more often reported for vortioxetine than the SSRIs. These AEs were also most prominent in the largest clusters found by the cluster analysis. Drug withdrawal syndrome, hyponatraemia, tremor and paraesthesia were less often reported for vortioxetine than the SSRIs. These AEs were seen in small clusters with fewer than ten reports (tremor [cluster 30], hyponatraemia [clusters 35 and 36]) or no cluster at all (drug withdrawal syndrome), except for paraesthesia (cluster 14).

\subsection{Strengths and Limitations}

A strength of this study is the use of global data for vortioxetine AEs and that the results of the clustering analysis mostly reflect the known safety profile for vortioxetine and SSRIs as a group. Clusters with symptoms relevant for current safety concerns could be selected for a case-level clinical review. When investigating a signal, other sources then spontaneous reports should be taken into account. This would indeed mean going back to clinical trial data to see if the event of interest was already reported there, possibly as separate event terms and not as a syndrome, and in addition conducting an in-depth literature search. However, fully investigating the signal potential of each cluster is beyond the scope of this study but could be done in a successive research.

The cluster analysis performed for the purpose of this study considered only reports with multiple AE terms. Reports with single AE terms carry no information on which AEs tend to occur in the same patient and there tends to exist a large number of reports with single AE terms that are identical. If included in the cluster analysis, they may have a disproportionate influence on the results. However, for a comprehensive overview of reporting patterns, reports with single AE terms can be retrospectively assigned to clusters obtained as described here.

Traditional methodology for statistical signal detection in pharmacovigilance often relies on disproportionality using a drug and an individual AE term. Often these methods investigate the relationship between a drug and separate AEs. With a cluster analysis, it is possible to identify natural groups of reports with similar patterns of AEs. One of the other advantages of this method is related to the coding of the AEs that takes place at national centre level. There are many possible ways to code the same AE with different separate coding terms. Seemingly diverse symptoms may relate to the same underlying condition or pathophysiology, which might be uncovered by a cluster analysis [10].
Syndromic surveillance through a cluster analysis for risperidone in VigiBase reliably recalled known disease spectra, while uncovering unexpected AE patterns requiring further review [29]. The method used in this study is based on the common mixture-model approach to a cluster analysis combined with consensus clustering, which was found to lead to stabler clusters with better clinical coherence [12].

\section{Conclusions}

A cluster analysis reveals a pattern of co-reported AEs that is consistent with labelled AEs for vortioxetine and the safety profile for SSRIs in general. In addition, clusters of unlabelled AEs were identified that reflect clinical entities and that might represent signals of previously unknown AEs. The VigiPoint analysis highlighted AEs that were more reported for vortioxetine than for SSRIs and these AEs were also seen in the main clusters identified in the cluster analysis. Novel analyses of spontaneous reports such as clustering may help to further understand the pathogenesis of AEs.

Supplementary Information The online version contains supplementary material available at https://doi.org/10.1007/s40264-021-01139-y.

\section{Declarations}

Funding No funding was received for the conduct of this research or the preparation of this article.

Conflicts of Interest/Competing Interests CE, FH, EP, RC, EM, HT and $\mathrm{NN}$ declare that they have no potential conflicts of interest that might be relevant to the contents of this manuscript.

Ethics Approval Not applicable.

Consent to Participate Not applicable.

Consent for Publication Not applicable.

Availability of Data and Material The VigiBase datasets generated and analysed during the current study are not publicly available because of agreements between contributors of data to the database used (VigiBase) and the custodian of this database. National centres (mainly national drug regulatory authorities) constituting the World Health Organization Programme for International Drug Monitoring contribute data to VigiBase, and the Uppsala Monitoring Centre is the custodian in its capacity as a World Health Organization collaborating centre for international drug monitoring.

Code Availability Not applicable.

Authors' Contributions CE, FH, EP, RC, EM, HT, NN: concept and design of the study. RC, EM, HT, NN: database strategy research and extraction of data. $\mathrm{CE}, \mathrm{FH}, \mathrm{EP}, \mathrm{RC}, \mathrm{EM}, \mathrm{HT}, \mathrm{NN}$ : data generation, collection, analysis and interpretation of data. All authors contributed to the writing/reviewing of the manuscript and approved the final version for submission. 
Open Access This article is licensed under a Creative Commons Attribution-NonCommercial 4.0 International License, which permits any non-commercial use, sharing, adaptation, distribution and reproduction in any medium or format, as long as you give appropriate credit to the original author(s) and the source, provide a link to the Creative Commons licence, and indicate if changes were made. The images or other third party material in this article are included in the article's Creative Commons licence, unless indicated otherwise in a credit line to the material. If material is not included in the article's Creative Commons licence and your intended use is not permitted by statutory regulation or exceeds the permitted use, you will need to obtain permission directly from the copyright holder. To view a copy of this licence, visit http://creativecommons.org/licenses/by-nc/4.0/.

\section{References}

1. Connolly KR, Thase ME. Vortioxetine: a new treatment for major depressive disorder. Expert Opin Pharmacother. 2016;17(3):421-31.

2. Schatzberg AF, Blier P, Culpepper L, Jain R, Papakostas GI, Thase ME. An overview of vortioxetine. J Clin Psychiatry. 2014;75(12):1411-8.

3. Yang K-C, Stepanov V, Amini N, Martinsson S, Takano A, Bundgaard $\mathrm{C}$, et al. Effect of clinically relevant doses of vortioxetine and citalopram on serotonergic PET markers in the nonhuman primate brain. Neuropsychopharmacology. 2019;44(10):1706-13.

4. Sanchez C, Asin KE, Artigas F. Vortioxetine, a novel antidepressant with multimodal activity: review of preclinical and clinical data. Pharmacol Ther. 2015;145:43-57.

5. Baldwin DS, Chrones L, Florea I, Nielsen R, Nomikos GG, Palo $\mathrm{W}$, et al. The safety and tolerability of vortioxetine: analysis of data from randomized placebo-controlled trials and open-label extension studies. J Psychopharmacol. 2016;30(3):242-52.

6. Carvalho AF, Sharma MS, Brunoni AR, Vieta E, Fava GA. The safety, tolerability and risks associated with the use of newer generation antidepressant drugs: a critical review of the literature. Psychother Psychosom. 2016;85(5):270-88.

7. Koesters M, Ostuzzi G, Guaiana G, Breilmann J, Barbui C. Vortioxetine for depression in adults. Cochrane Database Syst Rev. 2017;7(7): CD011520.

8. European Medicines Agency. Brintellix: summary of product characteristics. https://www.ema.europa.eu/en/documents/produ ct-information/brintellix-epar-product-information_en.pdf. Accessed 18 Dec 2021.

9. US FDA. Trintellix: summary of product characteristics. https:// www.accessdata.fda.gov/drugsatfda_docs/label/2017/204447s013 lbl.pdf. Accessed 18 Dec 2021.

10. Chandler RE, Juhlin K, Fransson J, Caster O, Edwards IR, Noren GN. Current safety concerns with human papillomavirus vaccine: a cluster analysis of reports in VigiBase ${ }^{\circledR}$. Drug Saf. 2017;40(1):81-90.

11. Erlanson N. Improving the speed and quality of an adverse event cluster analysis with stepwise expectation maximization and community detection. 2020. http://uu.diva-portal.org/smash/get/diva2: 1451279/FULLTEXT01.pdf. Accessed 18 Dec 2021.

12. Noren GN, Meldau EL, Chandler R. Consensus clustering for case series identification and adverse event profiles in pharmacovigilance. Artif Intell Med. 2021. https://doi.org/10. 1016/j.artmed.2021.102199.

13. Lindquist M. VigiBase, the WHO global ICSR database system: basic facts. Drug Inf J. 2008;42(5):409-19.

14. Lagerlund O, Strese S, Fladvad M, Lindquist M. WHODrug: a global, validated and updated dictionary for medicinal Iiformation. Ther Innov Regul Sci. 2020;54(5):1116-22.

15. Juhlin K, Star K, Noren GN. A method for data-driven exploration to pinpoint key features in medical data and facilitate expert review. Pharmacoepidemiol Drug Saf. 2017;26(10):1256-65.

16. Heiser JF, Wilcox CS. Serotonin 5-HT1a receptor agonists as antidepressants. CNS Drugs. 1998;10(5):343-53.

17. Meeusen R, Watson P, Hasegawa H, Roelands B, Piacentini MF. Central fatigue: the serotonin hypothesis and beyond. Sports Med. 2006;36(10):881-909.

18. Krakowski M. Violence and serotonin: influence of impulse control, affect regulation, and social functioning. J Neuropsychiatry Clin Neurosci. 2003;15(3):294-305.

19. Breggin PR. Suicidality, violence and mania caused by selective serotonin reuptake inhibitors (SSRIs): a review and analysis. Int J Risk Saf Med. 2003;16:31-49.

20. World Health Organization. Vortioxetine and aggression. WHO pharmaceuticals newsletter. https://apps.who.int/iris/bitstream/ handle/10665/329688/WPN-2019-05-eng.pdf?sequence=1\&isAll owed $=\mathrm{y}$. Accessed 18 Dec 2021.

21. European Medicines Agency. Scientific conclusions and grounds for the variation to the terms of the marketing authorisation(s). 2020. https://www.ema.europa.eu/en/documents/scientific-concl usion/brintellix-h-c-psusa-00010052-201909-epar-scientific-concl usions-grounds-variation-terms-marketing_en.pdf. Accessed 18 Dec 2021.

22. Geneesmiddeleninformatiebank. https://www.geneesmiddeleni nformatiebank.nl/nl/. Accessed 18 Dec 2021.

23. Praharaj SK. Serotonin reuptake inhibitor induced sensory disturbances. Br J Clin Pharmacol. 2004;58(6):673-4.

24. Arima Y, Kubo C, Tsujimoto M, Ohtani H, Sawada Y. Improvement of dry mouth by replacing paroxetine with fluvoxamine. Ann Pharmacother. 2005;39(3):567-71.

25. North Metropolitan Health Service. Ocular effects of serotonin antidepressants. Graylands Hosp Drug Bull. October 2016 Vol 23 No.2 ISSN 1323-1251.

26. Mansuy L. Antidepressant therapy with milnacipran and venlafaxine. Neuropsychiatr Dis Treat. 2010;6(Suppl. I):17-22.

27. van Hunsel F, de Waal S, Härmark L. The contribution of direct patient reported ADRs to drug safety signals in the Netherlands from 2010 to 2015. Pharmacoepidemiol Drug Saf. 2017;26(8):977-83.

28. Watson S, Chandler RE, Taavola H, Härmark L, Grundmark B, Zekarias A, et al. Safety concerns reported by patients identified in a collaborative signal detection workshop using VigiBase: results and reflections from Lareb and Uppsala Monitoring Centre. Drug Saf. 2018;41(2):203-12.

29. Noren GN, Fransson J, Juhlin K, Chandler R, Edwards IR. Adverse event cluster analysis for syndromic surveillance [abstract]. In: Proceedings of 15th ISoP annual meeting "Cubism in Pharmacovigilance" Prague, Czech Republic 27-30 October, 2015. Drug Saf. 2015;38(10):958. 